\title{
Effectiveness of electroacupuncture for thin endometrium in infertile women: study protocol for a single-blind, randomized controlled trial
}

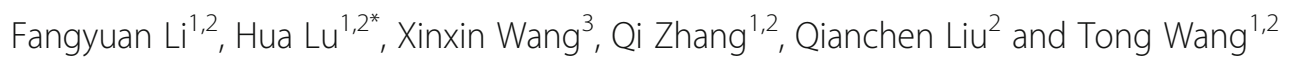

\begin{abstract}
Background: Thin endometrium negatively impacts the reproductive function. Current treatments for thin endometrium do not always improve endometrial receptivity. Preliminary evidence suggests that electroacupuncture could have potential therapy for thin endometrium in infertile women. Thus, this randomized controlled trial was designed to test whether electroacupuncture can improve endometrial receptivity in infertile women with thin endometrium.

Methods: This study is a randomized, single-blinded, controlled, clinical trial. A total of 142 eligible patients will be recruited and randomly assigned to the electroacupuncture (EA) group or the sham electroacupuncture (SEA) group in a 1:1 ratio. Participants will receive 36 sessions over three menstrual cycles (12 weeks in total), with the same acupoint prescription. The primary outcome of this trial is endometrial thickness in the midluteal phase. The secondary outcomes include endometrial pattern, resistance index (RI) and pulsatility index (PI) of bilateral uterine artery and endometrium blood flow, serum estradiol (E2) and progesterone (P), and pregnancy rate. The pregnancy rate will be evaluated during a 6-month follow-up after completion of the trial. All other outcomes will be evaluated before treatment, during the treatment of 1st, 2nd, and 3rd menstrual cycle, and 6 months after treatment.
\end{abstract}

Discussion: If the outcome confirms the effectiveness of electroacupuncture for thin endometrium in infertile women, this treatment will be proposed for application in clinical practice.

Trial registration: Chinese Clinical Trials Registry ChiCTR2000029983. Registered on 18 February 2020

Keywords: Electroacupuncture, Thin endometrium, Effectiveness, RCT

\footnotetext{
* Correspondence: Ih18980880525@126.com

${ }^{1}$ College of Clinical Medicine, Chengdu University of Traditional Chinese Medicine, NO.37 Shi-er-giao Road, Chengdu 610075, Sichuan Province, People's Republic of China

${ }^{2}$ Hospital of Chengdu University of Traditional Chinese Medicine, NO.39

Shi-er-qiao Road, Chengdu 610075, Sichuan Province, People's Republic of

China

Full list of author information is available at the end of the article
}

(c) The Author(s). 2021 Open Access This article is licensed under a Creative Commons Attribution 4.0 International License, which permits use, sharing, adaptation, distribution and reproduction in any medium or format, as long as you give appropriate credit to the original author(s) and the source, provide a link to the Creative Commons licence, and indicate if changes were made. The images or other third party material in this article are included in the article's Creative Commons licence, unless indicated otherwise in a credit line to the material. If material is not included in the article's Creative Commons licence and your intended use is not permitted by statutory regulation or exceeds the permitted use, you will need to obtain permission directly from the copyright holder. To view a copy of this licence, visit http://creativecommons.org/licenses/by/4.0/. The Creative Commons Public Domain Dedication waiver (http://creativecommons.org/publicdomain/zero/1.0/) applies to the data made available in this article, unless otherwise stated in a credit line to the data. 


\section{Background}

Previous research has shown that endometrial thickness is related to endometrial receptivity [1]. An inadequate endometrium can be considered as a main hindrance to fertility [2]. It has been shown that thin endometrium leads to infertility [3]. Likewise, a "thin" endometrium is associated with implantation failure and low implantation rates $[1,4]$. Globally, 8 to $12 \%$ of reproductive age couples suffer from infertility which has become a major global public health issue [5]. Despite the advances in assisted reproduction, many patients with thin endometrium repeated implantation failure [6]. It has been reported that nearly two-thirds of failed implantation in IVF (in vitro fertilization) are caused by poor endometrial receptivity [7]. Incidences of thin endometrium in ovarian stimulation cycles and natural cycles are $38-66 \%$ [8] and 5-25\% [9], respectively. In fresh or frozenthawed embryo transfer cycles, thin endometrium often leads to cancelation of ET and cryopreservation of all the embryos [10]. A thin endometrium is associated with low conception rate [11-13], but also to a higher probability of spontaneous abortion and ectopic pregnancy [14]. The probability of clinical pregnancy was $23.3 \%$ for an endometrium measuring $\leq 7 \mathrm{~mm}$, which was significantly lower than that of endometrium measuring $>7$ $\mathrm{mm}(48.1 \%)$ [13]. The lowest pregnancy rates were associated with endometrial thickness of $<7 \mathrm{~mm}$ in 768 consecutive medicated FER (frozen embryo replacement) cycles [15]. Hence, thin endometrium is often defined as less than $7 \mathrm{~mm}$ during the late follicular phase or after ovulation [16, 17]. Endometrial receptivity appears to be the bottleneck of the reproductive process [18]. Therefore, improving endometrial receptivity among infertile patients with thin endometrium is urgently needed in reproductive medicine.

The endometrium, a unique cyclic tissue regeneration system, depends on the cyclical growth and regression of blood vessels that supply the endometrium [19]. Previous works showed that angiogenesis which depends on the expression of angiogenic factors (VEGF, FGF, and angiopoietin families) is a critical factor in determining the endometrial receptivity during the implantation window (IW) [20]. Thin endometria are characterized by high uterine blood flow impedance, poor growth of glandular epithelium, decreased expression of vascular endothelial growth factor (VEGF), and poor vascular development [21]. Current therapeutic strategies for thin endometrium include long-term administration of exogenous estrogen, low-dose of aspirin, vaginal sildenafil citrate application, transvaginal endometrial perfusion of granulocyte colonystimulating factor (G-CSF), autologous platelet-rich plasma infusion (PRP), stem cell therapy, vitamins $C$ and $\mathrm{E}$, and L-arginine supplement $[8,22]$. Despite the vast diversity of treatment, most of these treatment options result in a minor change in the endometrium thickness and pregnancy rate [22]. Currently, there is no one acceptable approach to treat thin endometrium [23], thus the need for an alternative and complementary therapy for patients with thin endometrium.

Acupuncture may have a potential therapeutic effect on thin endometrium in infertile women. Pulsatility index (PI) in the uterine arteries is considered valuable in assessing endometrial receptivity, and it was found to have decreased after EA treatment [24]. EA has been found to improve endometrial angiogenesis during periimplantation period by increasing the expression of VEGF R2/PI3K/AKT and VEGFR2/ERK signaling pathways in $\mathrm{COH}$ rats [25]. Estrogen promotes the reconstruction of the endometrial vascular system and the repair of damaged endometrium by binding to the estrogen receptor (ER) ligand [26]. A significant increase in endometrial thickness and the number of endometrial glands after EA treatment, probably due to up-regulation of serum $\mathrm{E}$ levels, and different regulation of the sex steroid receptors $E R \alpha$ and ER $\beta$ [27]. EA also plays an effective auxiliary role of supporting bone marrow mesenchymal stem cell (BMSCs) in repair of thin endometrium [28]. Furthermore, acupuncture may ameliorate the uterine environment by increasing the glandular area and expression of receptivity markers such as LIF and OPN proteins [29]. Clinical studies have found that acupuncture may improve the high-quality embryo rate [30] and clinical pregnancy rate [31] by improving endometrial blood flow state and the uterine environment. Although acupuncture has initially been shown to improve endometrial receptivity and pregnancy rates during the process of assisted reproduction, the associated evidence base is poor [32]. Thus, well-designed RCT should be conducted based on a rigorous methodology to evaluate the efficacy of EA in improving endometrial receptivity for thin endometrium in infertile women.

\section{Methods/design \\ Study design}

A single-center, patient-blinded, randomized controlled trial (RCT) was devised following the Consolidated Standards of Reporting Trials (CONSORT) statement [33], the Standardized Protocol Items: Recommendations for Interventional Trials (SPIRIT) guidelines [34], and the Revised Standards for Reporting Interventions in Clinical Trials of Acupuncture (STRICTA) [35]. If participants are eligible and agree to participate, they will be randomly assigned to electroacupuncture group (EA) or sham-electroacupuncture (SEA) group in a 1:1 ratio. The trial will be conducted in the Department of gynecology, Chengdu University of Traditional Chinese Medicine Affiliated Hospital. The flowchart is shown in Fig. 1. 


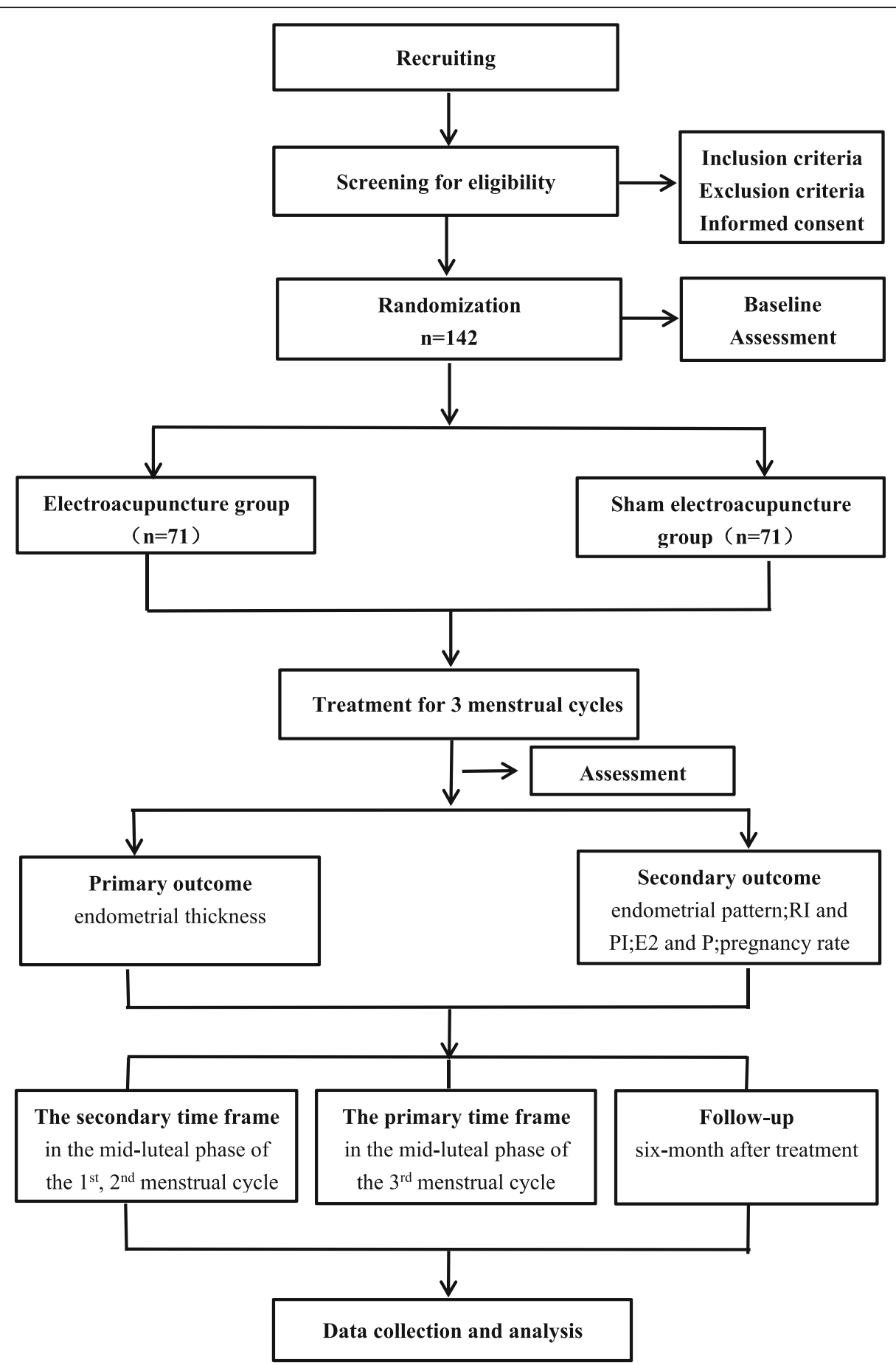

Fig. 1 Trial flow chart

\section{Ethics}

The trial will be carried out in accordance with the Declaration of Helsinki [36]. Ethical approval has been obtained from the Sichuan Regional Ethics Review Committee on Traditional Chinese Medicine with an ethics approval number of 2019KL-072. The work reported in this article is registered with an identifier
(ChiCTR2000029983) by the Chinese Clinical Trial Registry.

\section{Participants}

The diagnostic criteria for thin endometrium are based on data provided in our previous studies: endometrial thickness $(\mathrm{EMT})<7 \mathrm{~mm}$ in the mid-luteal phase (7 days 
after the day of ovulation or on day 21 of an unstimulated ovarian cycle) $[8,15,17]$. Infertility will be diagnosed as the failure to conceive for at least 12 months of unprotected sexual intercourse [37].

\section{Inclusion criteria}

Patients who conform to the following criteria will be included:

1. Females, aged between 20 and 45 years;

2. Meets the diagnosis of infertility and is trying to conceive;

3. EMT $<7 \mathrm{~mm}$ in the midluteal phase;

4. Willing to participate in this study and sign an informed consent form.

\section{Exclusion criteria}

1. Infertility induced by an ovulation disorder, severe endometriosis, Asherman's syndrome, luteal phase deficiency, fallopian tube obstruction, or male infertility;

2. Pregnancy or lactation;

3. History of psychiatric disorders (serious anxiety and depression, schizophrenia), coagulation disorders, blood infections (viral hepatitis, HIV), malignant tumor, diabetic neuropathy or complicated with serious heart, kidney or liver disease;

4. A cardiac pacemaker, metal allergy, or artificial joint;

5. History of acupuncture treatment in the last 3 months or use of estrogen, progestin in the past 4 weeks;

6. Participation in other clinical trials within the past 3 months.

\section{Dropout criteria}

Patients who quit the study voluntarily or have poor clinical compliance during observation period or found to not conform to the inclusion criteria after enrolment will be considered as having dropped out.

\section{Recruitment strategies}

Infertile female participants with thin endometrium will be recruited either through advertisements on notice boards in Chengdu University of Traditional Chinese Medicine Affiliated Hospital or advertisements in hospital social media (WeChat). Any interested patients will reach the researcher by telephone, email, or WeChat. If they meet the study criteria, they will be invited for a face-to-face interview for baseline screening with the researcher. The researcher will explain the study in detail, including potential benefits and risks. Once a participant agrees to participate in the study, informed consent will be obtained before randomization. Participants consent forms is shown in Additional file 1.

\section{Randomization}

The participants will be randomly assigned to electroacupuncture group or sham-electroacupuncture group in a 1:1 ratio. Randomization will be performed based on a random list of numbers generated by SPSS21.0 software (International Business Machines Corp., Armonk, NY). Allocation will be conducted by a designated researcher who has no contact with any participant and is not involved in the data collection or analysis. Allocation will be concealed in sequentially numbered, opaque, and sealed envelopes containing the randomization assignments. The appropriate numbered, opaque, sealed envelope will be opened after each participant meets eligibility criteria and signs informed consent. The allocation concealment to the doctors is not feasible for trials on acupuncture. The acupuncturist will not be allowed to reveal any information about treatment procedures and outcomes to the participants or the assessor.

\section{Blinding}

The specific nature of the intervention makes it impossible to blind the acupuncturist. However, the participants, data collection staff, and data analysts will be blinded. To maximize blinding of participants, placebo needles and sham electroacupuncture design will be used in the sham-electroacupuncture group. Adhesive pads will be applied to both EA and SEA groups to ensure that the participants are unaware of the differences between the two acupuncture treatments. Each participant will receive intervention in a personal space separated by bed curtain and no communication will be allowed between participants. During the intervention period, the acupuncturist and the data collection researcher will not be allowed to exchange information on grouping, treatment procedures, and outcomes. Blinded evaluation and blinded statistical analysis will be emphasized during the data collection and analysis stage.

\section{Interventions}

Study flow

The treatments will be performed by one licensed acupuncturist who has at least 5 years of experience with acupuncture. All participants will be treated in supine position on the treatment couch. Treatment will be conducted during three menstrual cycles, at a frequency of 3 sessions per week (once every 2-3 days) in both intervention and control groups after randomization with each group receiving $30 \mathrm{~min}$ per session. If a woman becomes pregnant during the intervention phase, the intervention will be terminated. Six months after completion 
of the treatment, follow-up will be performed by telephone or WeChat to ask if the participants are pregnant.

\section{Electroacupuncture (EA) group}

To improve endometrial receptivity in infertile patients with thin endometrium, electroacupuncture prescription will be developed based on clinical practice and literature review. The obligatory acupoints will include bilateral Tianshu (ST25), Zigong (EX-CA1), Sanyinjiao (SP6), Guilai (ST29), individual Zhongji (RN3), and Guanyuan (RN4). Additionally, sets of adjunct acupoints will be alternately selected by the acupuncturists based on the periodic therapy of Traditional Chinese Medicine: bilateral Xuehai (SP10) and Diji (SP8) will be needled during menstruation; bilateral Taichong (LR3) and Taixi (KI3) will be needled after menstruation; bilateral Taichong (LR3), Hegu (LI4), and Zusanli (ST36) will be needled during the ovulatory period; individual Qihai (RN6) and bilateral Zusanli (ST36) will be needled before the next menstruation. Acupuncture points will be identified according to the method of point location issued by the World Health Organization. Stick adhesive pads (15 $\mathrm{mm}$ ) will be placed at all points. After sterilization of the skin with alcohol wipes, single-use, stainless, sterile needles (gauge $0.25 \times 40 \mathrm{~mm}$ or $0.25 \times 60 \mathrm{~mm}$; Hwato, Suzhou, China) will be needle inserted.

In a supine position, the needle will be inserted $40 \mathrm{~mm}$ vertically into the muscles of the abdominal wall through the pads for ST25, ST29, RN4, and RN3. EX-CA1 and RN6 will be inserted vertically to a depth of $25 \mathrm{~mm}$ in the abdomen. The needle will then be inserted vertically at SP6, SP10, and SP8 to a depth of $25 \mathrm{~mm}$. LR3 will be punctured vertically to a depth of $15 \mathrm{~mm}$ into the skin, while LI4 and ST36 will be punctured perpendicularly to a respective depth of $25 \mathrm{~mm}$ and $40 \mathrm{~mm}$, respectively. Deqi sensation (including numbness, soreness, distention, heaviness) will be achieved through lifting, thrusting, and rotating for $10 \mathrm{~s}$ on each point. An electric stimulator (HANS-200E acupoint nerve stimulator, Nanjing Jisheng Medical Co, Ltd) will be applied to bilateral ST25 and ST29 with disperse-dense waves of $2 / 100 \mathrm{~Hz}$ frequencies for $30 \mathrm{~min}$. The current intensity will be increased until the needles start vibrating slightly. After a 30-min retention period, all needles, electrode clamps, and stick adhesive pads will be removed.

\section{Sham-electroacupuncture (SEA) group}

Participants in the sham electroacupuncture group will receive sham EA on therapeutic acupoints without skin penetration or electrical stimulation [38]. The procedures, frequency, and duration of treatment will be the same as that of the electroacupuncture group. Blunt needles which are similar to Streitberger needles device will be used [39]. This type of needle can provoke a needling sensation quite similar to real acupuncture as soon as it touches the skin, without insertion. The acupuncturists should pretend to manipulate the needles slightly with an even lifting, thrusting, and twisting method for $10 \mathrm{~s}$ on each point as well. No Deqi sensation will be induced. Similarly, the needles on bilateral ST25 and ST29 will be connected to a sham electric stimulator with the same parameters as the EA group, but the wire will be cut thus no current intensity. The light seen and the sound of the pulse generator heard will be the same as the EA group.

\section{Outcome measures \\ Primary outcome}

The primary outcome will be endometrial thickness in the mid-luteal phase of the 3rd menstrual cycle (the primary time frame), the 1st menstrual cycle and 2nd menstrual cycle as well as 6 months after completion of the treatment (the secondary time frame) compared with baseline (in the -1 st menstrual cycle).

\section{Secondary outcome measures}

The secondary outcomes include the following four aspects: (1) endometrial pattern, (2) RI and PI of bilateral uterine artery and endometrium blood flow, (3) serum estradiol (E2) and progesterone (P), and (4) pregnancy rate. The first three of the secondary outcomes will be evaluated in the mid-luteal phase of 3rd menstrual cycle (the primary time frame) and the 1st menstrual cycle and 2nd menstrual cycle, as well as 6 months after treatment (the secondary time frame) in comparison with the baseline (in the -1 st menstrual cycle). The pregnancy rate will be assessed during a 6-month follow-up after completion of the trial.

A certified sonographer will measure the endometrial thickness and the first three secondary outcomes through transvaginal ultrasound. A laboratory physician will test the serum estradiol and progesterone through radioimmunoassay. They will be trained and assessed before the trial.

\section{Assessment of adverse events}

Risks associated with this study are minimal. However, some possible adverse events may include bleeding, hematoma, fainting, continuous post needling pain, local infection, and other symptoms. Participants will be asked to report any adverse events during the trial, and these will be recorded on an adverse event report form in the case report form (CRF). Once a serious adverse event occurs, the researcher will terminate the trial and rescue procedures will be initiated at once. A written report to the Ethics Committee should be submitted immediately. The overview of the outcome measurement at different time points is shown in Table 1. 
Table 1 Data collection points

\begin{tabular}{|c|c|c|c|c|c|c|}
\hline & Baseline & & \multicolumn{3}{|c|}{ Treatment } & \multirow{2}{*}{$\begin{array}{c}\text { Follow-up } \\
\text { (6) }\end{array}$} \\
\hline & (1) & (2) & (3) & (4) & (5) & \\
\hline Time point & $-1 \mathrm{st} \mathrm{MC}$ & 0 & $1 \mathrm{st} \mathrm{MC}$ & 2nd MC & 3rd MC & $\begin{array}{c}6 \text { months after } \\
\text { treatment }\end{array}$ \\
\hline \multicolumn{7}{|l|}{ Enrolment } \\
\hline Age & $x$ & & & & & \\
\hline Duration of disease & $x$ & & & & & \\
\hline Disease history & $x$ & & & & & \\
\hline Comorbidity & $x$ & $x$ & $x$ & $x$ & $x$ & $x$ \\
\hline $\begin{array}{l}\text { Concomitant } \\
\text { medication }\end{array}$ & $x$ & & & & & $x$ \\
\hline $\begin{array}{l}\text { Inclusion/Exclusion } \\
\text { criteria }\end{array}$ & $x$ & & & & & \\
\hline $\begin{array}{c}\text { Informed consent } \\
\text { signed }\end{array}$ & $x$ & & & & & \\
\hline $\begin{array}{l}\text { Randomization and } \\
\text { blinding }\end{array}$ & $x$ & & & & & \\
\hline \multicolumn{7}{|l|}{ Intervention } \\
\hline \multirow{2}{*}{\multicolumn{7}{|c|}{$\begin{array}{l}\text { Electro-acupuncture } \\
\text { (EA) }\end{array}$}} \\
\hline & & & & & & \\
\hline \multicolumn{7}{|l|}{$\begin{array}{c}\text { Sham } \\
\text { electro-acupuncture } \\
\text { (SEA) }\end{array}$} \\
\hline \multicolumn{7}{|l|}{ Assessment } \\
\hline $\begin{array}{l}\text { endometrial } \\
\text { thickness }\end{array}$ & $x$ & & $x$ & $x$ & $x$ & $x$ \\
\hline endometrial pattern & $x$ & & $x$ & $x$ & $x$ & $x$ \\
\hline RI and PI & $x$ & & $x$ & $x$ & $x$ & $x$ \\
\hline Serum estradiol & $x$ & & $x$ & $x$ & $x$ & $x$ \\
\hline progesterone & $x$ & & $x$ & $x$ & $x$ & $x$ \\
\hline pregnancy rate & & & & & & $x$ \\
\hline \multicolumn{7}{|l|}{$\begin{array}{c}\text { Data collection and } \\
\text { statistical analysis }\end{array}$} \\
\hline Adverse events & & & $x$ & $x$ & $x$ & $x$ \\
\hline Causes of dropout & & & $x$ & $x$ & $x$ & $x$ \\
\hline Compliance analysis & & & & & & $x$ \\
\hline Data analysis & & & & & & $x$ \\
\hline
\end{tabular}




\section{Sample size}

There is insufficient previous data on endometrial thickness after treatment with EA in infertile women with thin endometrium. Besides, the effect of acupuncture varies with different acupoint selection. The aim of the research was to clarify the effects of EA on thin endometrium in infertile women. Therefore, the change in endometrial thickness will be used as an evaluation index. Based on our pilot study, changes in endometrial thickness of the mid-luteal phase after a 12-week treatment were shown to be $8.30 \pm 2.83 \mathrm{~mm}(n=10)$ in the electroacupuncture group (EA) and $7.10 \pm 1.97 \mathrm{~mm}(n=$ 9) in the sham electroacupuncture group (SEA). Measurement data of parallel control trials (1:1) will be analyzed statistically using the following formula for sample size estimation:

$$
n=\frac{\left(Z_{\alpha}+Z_{\beta}\right)^{2} * 2 \sigma^{2}}{\delta^{2}}
$$

With a $5 \%$ significance level $(\alpha=0.05$, two-sided $)$ and $80 \%$ power $(\beta=0.2)$, at least 62 patients should be enrolled in each group and a total of 142 participants will be recruited to allow for a $15 \%$ dropout rate.

\section{Data management and monitoring}

This study will use four levels of data monitoring to improve quality control. A quality controller will check the relevant data in detail on the original CRFs and medical records regularly. The supervision's main content is whether the initial medical history is accurate, whether the CRF form is real, and whether the correction is standardized. Supervisors will audit the accuracy of registered information, data entry, and outcomes. Any errors required that the audit be repeated from the beginning. The principal investigator will evaluate whether the recruitment and intervention procedures are conducted with rigorous adherence to the protocol. An independent data monitoring committee (DMC) consisting of three experts (an epidemiologist, an acupuncture expert, and a gynecologist) will monitor and evaluate the recruitment of subjects, the progress of research implementation, and the data's safety, confidentiality, and integrity monthly. DMC will also regularly submit audit reports to the project management department and provide recommendations to continue the clinical trial, modify the trial protocol, or terminate the trial.

\section{Statistical analysis}

Statistical analyses of data will be performed using SPSS21.0 software (International Business Machines Corp., Armonk, NY) by analysts who will be blinded to the patients' allocation. To evaluate the curative effect in this trial, an intention-to-treat analysis (ITT) will be performed. Categorical variables will be presented by frequency (percentage) and analyzed with the chisquared test or Fisher's exact test. Continuous variables will be presented as mean \pm standard deviation $(M \pm S D)$ if they meet normal distribution. Otherwise, they will be presented as medians \pm interquartile range $(M \pm I Q R)$. The demographic characteristics will be compared between the groups by independent $t$ tests at baseline. All longitudinal analyses for continuous variables measured at baseline and three-time periods will be performed using linear mixed modeling. Comparisons between the intervention and control groups will be performed using the chi-square test or Fisher s exact test, as appropriate, for categorical variables. To evaluate the safety of acupuncture, we will use a Fisher exact test to report the relative risk of an adverse effect. All the tests will be two-sided, and a $P$ value of less than 0.05 will be considered to be statistically significant.

\section{Quality control and trial monitoring}

To guarantee the quality of the study, all researchers especially the acupuncturists will attend a series of training courses. The training includes how to use the randomization method, fill the case report form, manipulate interventions correctly, and other details of the trial. They will be required to master all of the details of the trial and pass a test before performing it. SOPs will be provided to practitioners to ensure identical treatment procedures. Acupuncturists who have been certified by the Ministry of Health of the People's Republic of China and have more than 5 years of clinical experience will be recruited to perform the procedures on the participants in the study.

Other therapies that may affect the endometrial thickness and receptivity will be prohibited during treatment, such as exogenous estrogen, low-dose of aspirin, and vaginal sildenafil citrate application. Any treatment information for other diseases should be recorded in detail. To improve compliance of the participants, the principle of voluntary participation, a treatment record sheet that facilitates real-time dynamic management, traffic compensation, and pregnancy knowledge education will be considered. Participants in the SEA group may get 36 sessions of electroacupuncture free of charge after the trial.

\section{Discussion}

\section{Shortcoming of other therapies}

Although there are many current treatment strategies for thin endometrium, they have some unsatisfactory deficiencies and lack sufficient evidence of efficacy. Exogenous estrogen treatment is the most widely used and convenient method [10]; the risk of estrogen therapy in promoting endometrial and breast neoplasms has been clarified over the past 3 to 4 decades [40, 41]. In 
addition, a clinical trial revealed that estradiol supplementation starting on the human chorionic gonadotropin (HCG) day for the patients with thin endometrium did not provide any benefit on the pregnancy outcome in intracytoplasmic sperm injection (ICSI) cycles [42]. Aspirin therapy has not resulted in an improvement in the endometrial thickness, and the resistance of uterine and ovarian flow [43, 44]. Although previous data suggest that G-CSF intrauterine infusion may improve endometrial thickness, no controlled studies have demonstrated that this approach improves clinical pregnancy rates or live birth rates. Moreover, the associated potential hazards or risks need to be further considered $[45,46]$. The use of stem cells appears to be a very promising option for the most refractory case, such as Asherman syndrome [47]. However, stem cell therapies are in their relative infancy [45], and more research is needed to evaluate the safety, effectiveness, and cost of this modality before it can be integrated as a medical treatment for this condition [46]. A previous trial showed that PRP efficiently improved the endometrium proliferation, implantation rate, and clinical pregnancy rate [48]; however, clinical trial data is limited, with the majority being non-randomized trials [49]. A systematic review indicates that only a few randomized controlled trials support the use of sildenafil in frozen embryo transfer (ET) for enhancing endometrial growth [50]. Other treatments, such as vitamin $\mathrm{E}$ and l-arginine, were all base on small sample size, thus lack sufficient evidence of efficacy [51].

\section{Outcome measures selection}

Endometrial receptivity plays a vital role in embryoendometrium cross talk. Endometrium exhibits a short period of receptivity, known as the "window of implantation" [2]. The IW appears in the mid-secretive phase of endometrium on the 20th to 23rd day in the menstrual cycle (namely the 7th-9th day after ovulation) [52]. Therefore, markers of endometrial receptivity are tested in the mid-luteal phase ( 7 days after the day of ovulation) for a natural menstrual cycle in clinical practice. The ultrasound markers for evaluation of endometrial receptivity are endometrial thickness, pattern, and blood flow impedance [5]. Endometrial thickness as an essential factor in endometrial receptivity is the most extensively used method in clinical practice [53, 54]. Previous research showed a positive correlation between endometrial thickness and implantation rates in 465 cycles of oocyte donation [55]. Endometrial blood perfusion is an important factor for endometrial growth [56]. Resistance and pulsatility index of subendometrial and uterine artery are useful tools for clinicians in predicting endometrial receptivity [57]. High blood flow impedance of uterine radial arteries is associated with poor endometrial growth in patients with thin endometrium [21]. The diastolic flow of the uterine artery in the midluteal phase is also associated with reproductive success [58]. Endometrial blood flow directly reflects the microenvironment of embryo implantation. Successful intrauterine implantation is positively associated with lower subendometrial resistance index (RI) and pulsatility index (PI) [59]. In the study, endometrial blood perfusion will be assessed using the PI and RI of bilateral uterine artery as well as endometrium blood flow. The endometrial patterns including type A (triple-line), type $\mathrm{B}$, and type $\mathrm{C}$ is classified according to morphology of the endometrium [60]. An earlier study showed that the presence of a triple-line endometrium with a thickness $>6 \mathrm{~mm}$ was strongly correlated with conception [61]. The triple line pattern assessed on the day of HCG injection OR IUI was associated with higher clinical pregnancy rates [54]. The endometrium undergoes complex changes in the circulation of estrogen and progesterone (P), which culminate at the mid-luteal phase of the menstrual cycle when it becomes receptive $[2,62,63]$. Serum estradiol and progesterone concentrations may reflect endometrial status [64]. Estradiol stimulates both increases in the size and number of myometrial and endometrial cells as well as changes in endometrial thickness [64]. It is noteworthy that there is a minimum concentration of progesterone which is required to induce normal secretory endometrial development and receptivity [65]. This is supported by a previous study which revealed a significant decrease in clinical pregnancy and live birth rate with serum progesterone level $<10 \mathrm{ng} / \mathrm{ml}$ [66].

\section{The selected acupoints}

In this trial, acupoints selection follows TCM theory or published articles. According to TCM theory, thin endometrium in infertile women combines with the deficiency of kidney essence, poor circulation of Qi and blood, and disharmony of thoroughfare and conception vessels. Based on the theory of acupuncture, RN3, RN4, and RN6 are located in the lower abdomen of the Ren meridian, which means dominating pregnancy, tonifying original Qi and strengthening the kidney. LR3 combines with LI4, which are called Si-guan points to regulate Qi circulation. SP6 is considered a classic acupoint for curing gynecological diseases. ST36 can facilitate the production of "qi" and "blood," while KI3 can nourish yin and kidney essence. Currently known, acupuncture mainly improves the endometrial receptivity by increasing endometrial blood perfusion or regulating hormone receptors [27, 32, 67]. Previous study indicated that stimulation of SP6 and ST36 by electroacupuncture improved implantation rate significantly in $\mathrm{COH}$ rats, which may indirectly influence the secretion of estrogen and progesterone as well as facilitate the endometrial 
angiogenesis [25]. Another study revealed that transcutaneous electrical acupuncture point stimulation (TEAS) at RN3 (CV3), RN4 (CV4), SP6, and EX-CA1 could have beneficial effects on ultrasound markers of endometrial receptivity [67]. EA at LR3, SP6, ST28, EX-CA1, RN6, and RN4 was able to reduce uterine artery blood flow impedance in patients undergoing in vitro fertilization [68]. Warm needling at ST25, CV4, CV3, EX-CA1, and ST36 could regulate endometrial morphology and blood flow to improve endometrial receptivity, embryo transplantation rate, and pregnancy rate as well as decrease early abortion rate [69]. Warm acupuncture may promote the clinical pregnancy rate by improving endometrial blood perfusion and endometrial receptivity at the points of Qihai (CV6), Guanyuan (CV4), Zhongji (CV3), Guilai (ST29), Zigong (EX-CA1), Zusanli (ST36), and Sanyinjiao (SP6) [31]. Finally, SP10, SP8, ST29, and EXCA1 are recommended to promote embryo implantation by consensus among experts in China [69].

This study has some limitations. Firstly, the acupuncturists cannot be blinded due to the nature of the intervention. Secondly, estimation of sample size of the proposed study was based on our previous clinical observation of a small sample size. Nevertheless, the results from this trial will provide evidence of EA as a possible therapy for thin endometrium in infertile women.

\section{Trial status}

The recruitment of patients will start on March 1, 2020, and it is expected that by December 31, 2020, the required sample size will be reached. This is the first version, and the protocol was approved on 18 February 2020.

\section{Supplementary Information}

The online version contains supplementary material available at https://doi. org/10.1186/s13063-021-05029-7.

\section{Additional file 1.}

\section{Abbreviations}

EA: Electroacupuncture; SEA: Sham electroacupuncture; RI: Resistance index; PI: Pulsatility index; E2: Estradiol; P: Progesterone; RCT: Randomized controlled trial; IVF: In vitro fertilization; FER: Frozen embryo replacement; ICSI: Intracytoplasmic sperm injection; HCG: Human chorionic gonadotropin; ET: Embryo transfer; ER: Estrogen receptor; VEGF: Vascular endothelial growth factor; BMSCs: Bone marrow mesenchymal stem cell;

CONSORT: Consolidated Standards of Reporting Trials; SPIRIT: Standardized Protocol Items: Recommendations for Interventional Trials; STRI CTA: Standards for Reporting Interventions in Clinical Trials of Acupuncture; EMT: Endometrial thickness; HIV: Human immunodeficiency virus; TCM: Traditional Chinese Medicine; CRF: Case report form; DMC: Data monitoring committee; ITT: Intention-to-treat; $\mathrm{M} \pm \mathrm{SD}$ : Mean \pm standard deviation; $M \pm I Q R$ : Medians \pm interquartile range; SOPs: Standard operating procedures; HPG: Hypothalamic-pituitary-gonad; LIF: Leukemia-inhibitor factor; OPN: Osteopontin; IW: Implantation window; $\mathrm{COH}$ : Controlled ovarian hyperstimulation; IUI: Intra-uterine insemination; TEAS: Transcutaneous electrical acupuncture point stimulation; MC: Menstrual cycle

\section{Acknowledgements}

Not applicable.

\section{Dissemination}

Study results will be presented at national and international scientific meetings and be published in peer-reviewed and clinical journals.

\section{Ancillary and post-trial care}

Risks associated with the intervention and control measures in this study are minimal. We will provide patients with information of pregnancy preparation and traffic compensation.

\section{Authors' contributions}

FYL contributed to the conception, design, and manuscript writing. $\mathrm{HL}$ contributed to the conception and final approval of the manuscript. XXW, QZ, QCL, and TW participated in the design and drafted the manuscript. All authors read and approved the final manuscript.

\section{Funding}

This trial was financially supported by funds from the Ministry of Science and Technology of the People's Republic of China (grant number 2018YFC1704305)

\section{Availability of data and materials}

Data sharing is not applicable to this article as no datasets were analyzed during the current study. During the trial, all patient data will be accessible only to the research team.

\section{Ethics approval and consent to participate}

Ethical approval has been obtained from the Sichuan Regional Ethics Review Committee on Traditional Chinese Medicine (an ethics approval number of 2019KL-072). Informed consent of all participants will be obtained. Important protocol modifications (e.g., changes to eligibility criteria, analyses, outcomes) will be reported to the Ethics Review Committee.

\section{Competing interests}

The authors declare that they have no competing interests.

\section{Author details}

${ }^{1}$ College of Clinical Medicine, Chengdu University of Traditional Chinese Medicine, NO.37 Shi-er-qiao Road, Chengdu 610075, Sichuan Province, People's Republic of China. ${ }^{2}$ Hospital of Chengdu University of Traditional Chinese Medicine, NO.39 Shi-er-qiao Road, Chengdu 610075, Sichuan Province, People's Republic of China. ${ }^{3}$ College of Acupuncture and Tuina, Chengdu University of Traditional Chinese Medicine, NO.37 Shi-er-qiao Road, Chengdu 610075, Sichuan Province, People's Republic of China.

Received: 11 July 2020 Accepted: 6 January 2021

Published online: 21 January 2021

\section{References}

1. Richter KS, Bugge KR, Bromer JG, Levy MJ. Relationship between endometrial thickness and embryo implantation, based on 1,294 cycles of in vitro fertilization with transfer of two blastocyst-stage embryos. Fertil Steril. 2007;87:53-9.

2. Strowitzki T, Germeyer A, Popovici R, von Wolff $M$. The human endometrium as a fertility-determining factor. Hum Reprod Update. 2006;12: 617-30.

3. Alfer J, Happel L, Dittrich R, Beckmann MW, Hartmann A, Gaumann A, et al. Insufficient angiogenesis: cause of abnormally thin endometrium in subfertile patients? Geburtshilfe Frauenheilkd. 2017;77(7):756-64.

4. Abdalla HI, Brooks AA, Johnson MR, Kirkland A, Thomas A, Studd JW. Endometrial thickness: a predictor of implantation in ovum recipients? Hum Reprod. 1994;9:363-5.

5. Inhorn MC, Patrizio P. Infertility around the globe: new thinking on gender, reproductive technologies and global movements in the 21 st century. Hum Reprod Update. 2015;21:411-26.

6. Margalioth EJ, Ben-Chetrit A, Gal M, Eldar-Geva T. Investigation and treatment of repeated implantation failure following IVF-ET. Hum Reprod. 2006;21:3036-43. 
7. Kunicki M, Łukaszuk K, Woclawek-Potocka I, Liss J, Kulwikowska P, Szczyptańska J. Evaluation of granulocyte colony-stimulating factor effects on treatment-resistant thin endometrium in women undergoing in vitro fertilization. Biomed Res Int. 2014;2014:913235.

8. Liu KE, Hartman M, Hartman A. Management of thin endometrium in assisted reproduction: a clinical practice guideline from the Canadian Fertility and Andrology Society. Reprod BioMed Online. 2019;39:49-62.

9. Sher G, Herbert C, Maassarani G, Jacobs MH. Assessment of the late proliferative phase endometrium by ultrasonography in patients undergoing in-vitro fertilization and embryo transfer (IVF/ET). Hum Reprod. $1991 ; 6: 232-7$

10. Liu SM, Zhou YZ, Wang HB, Sun ZY, Zhen JR, Shen K, et al. Factors associated with effectiveness of treatment and reproductive outcomes in patients with thin endometrium undergoing estrogen treatment. Chin Med J. 2015;128(23):3173-7.

11. Wu Y, Gao X, Lu X, Xi J, Jiang S, Sun Y, et al. Endometrial thickness affects the outcome of in vitro fertilization and embryo transfer in normal responders after GnRH antagonist administration. Reprod Biol Endocrinol. 2014;12:96.

12. Lu YC, Yang J, Ding GL, Shi S, Zhang D, Jin L, et al. Small-conductance, calcium-activated potassium channel 3 (SK3) is a modulator of endometrial remodeling during endometrial growth. J Clin Endocrinol Metab. 2014;99: 3800-10.

13. Kasius A, Smit JG, Torrance HL, Eijkemans MJ, Mol BW, Opmeer BC, et al. Endometrial thickness and pregnancy rates after IVF: a systematic review and meta-analysis. Hum Reprod Update. 2014;20:530-41.

14. Yuan X, Saravelos SH, Wang Q, Xu Y, Li TC, Zhou C. Endometrial thickness as a predictor of pregnancy outcomes in 10787 fresh IVF-ICSI cycles. Reprod BioMed Online. 2016;33:197-205.

15. El-Toukhy T, Coomarasamy A, Khairy M, Sunkara K, Seed $P$, Khalaf $Y$, et al. The relationship between endometrial thickness and outcome of medicated frozen embryo replacement cycles. Fertil Steril. 2008;89:832-9.

16. Sharma S, Rani G, Bose G, Saha I, Bathwal S, Chakravarty BN. Tamoxifen is better than low-dose clomiphene or gonadotropins in women with thin endometrium ( $<7 \mathrm{~mm}$ ) after clomiphene in intrauterine insemination cycles: a prospective study. J Hum Reprod Sci. 2018;11(1):34-9.

17. Kumbak B, Erden HF, Tosun S, Akbas H, Ulug U, Bahçeci M. Outcome of assisted reproduction treatment in patients with endometrial thickness less than $7 \mathrm{~mm}$. Reprod BioMed Online. 2009;18:79-84.

18. Revel A. Defective endometrial receptivity. Fertil Steril. 2012;97:1028-32.

19. Jiang $P$, Tang $X$, Wang $H$, Dai $C$, Su J, Zhu H, et al. Collagen-binding basic fibroblast growth factor improves functional remodeling of scarred endometrium in uterine infertile women: a pilot study. Sci China Life Sci. 2019;62:1617-29.

20. Rizov M, Andreeva P, Dimova I. Molecular regulation and role of angiogenesis in reproduction. Taiwan J Obstet Gynecol. 2017;56(2):127-32

21. Miwa I, Tamura H, Takasaki A, Yamagata Y, Shimamura K, Sugino N. Pathophysiologic features of "thin" endometrium. Fertil Steril. 2009;91:9981004.

22. Lebovitz O, Orvieto R. Treating patients with "thin" endometrium - an ongoing challenge. Gynecol Endocrinol. 2014;30:409-14.

23. Mouhayar Y, Franasiak JM, Sharara Fl. Obstetrical complications of thin endometrium in assisted reproductive technologies: a systematic review. J Assist Reprod Genet. 2019;36:607-11.

24. Stener-Victorin $E$, Waldenström $U$, Andersson SA, Wikland M. Reduction of blood flow impedance in the uterine arteries of infertile women with electro-acupuncture. Hum Reprod. 1996;11:1314-7.

25. Chen W, Chen J, Xu M, Zhong Z, Zhang Q, Yang W, et al. Electroacupuncture facilitates implantation by enhancing endometrial angiogenesis in a rat model of ovarian hyperstimulation. Biol Reprod. 2019; 100(1):268-80

26. Zadehmodarres S, Salehpour S, Saharkhiz N, Nazari L. Treatment of thin endometrium with autologous platelet-rich plasma: a pilot study. JBRA Assist Reprod. 2017;21:54-6.

27. Ma S, Li D, Feng Y, Jiang J, Shen B. Effects of electroacupuncture on uterine morphology and expression of oestrogen receptors in ovariectomised rats. Acupunct Med. 2017;35:208-14

28. Xia L, Meng Q, Xi J, Han Q, Cheng J, Shen J, et al. The synergistic effect of electroacupuncture and bone mesenchymal stem cell transplantation on repairing thin endometrial injury in rats. Stem Cell Res Ther. 2019;10(1):244.
29. Fu H, He Y, Gao Y, Man Y, Liu W, Hao H. Acupuncture on the endometrial morphology, the serum estradiol and progesterone levels, and the expression of endometrial leukaemia-inhibitor factor and osteopontin in rats. Evid Based Complement Alternat Med. 2011;2011:606514. https://doi. org/10.1155/2011/606514.

30. Chen Q, Hau C. Impacts on pregnancy outcome treated with acupuncture and moxibustion in IVF-ET patients. Zhongguo Zhen Jiu. 2015;35(4):313-7.

31. Su WW, Gao XA, Gao ZY, Pan JL, Deng F, He WT. Clinical observation on the therapeutic effect of warm acupuncture on endometrial cavity fluid from in vitro fertilization-embryo transfer. Zhongguo Zhen Jiu. 2019;39(9):923-6.

32. Zhong Y, Zeng F, Liu W, Ma J, Guan Y, Song Y. Acupuncture in improving endometrial receptivity: a systematic review and meta-analysis. BMC Complement Altern Med. 2019;19(1):61.

33. Schulz KF, Altman DG, Moher D, CONSORT Group. CONSORT 2010 statement: updated guidelines for reporting parallel group randomised trials. PLoS Med. 2010;7(3):e1000251.

34. Chan AW, Tetzlaff JM, Altman DG, Laupacis A, Gøtzsche PC, Krle A-Jerić K, et al. SPIRIT 2013 statement: defining standard protocol items for clinical trials. Ann Intern Med. 2013;158(3):200-7.

35. MacPherson H, Altman DG, Hammerschlag R, Youping L, Taixiang W, White A, et al. Revised STandards for Reporting Interventions in Clinical Trials of Acupuncture (STRICTA): extending the CONSORT statement. Aust J Acupunct Chinese Med. 2010;7(6):35-46.

36. Idänpään-Heikkilä JE. Ethical principles for the guidance of physicians in medical research--the Declaration of Helsinki. Bull World Health Organ. 2001;79(4):279.

37. Rowe PJ, Comhaire FH, Hargreave TB. World Health Organization manual for the standardized investigation and diagnosis of the infertile couple. New York: Cambridge University Press; 2000. p. 6.

38. Chen ZX, Li Y, Zhang XG, Chen S, Yang WT, Zheng XW, et al. Sham electroacupuncture methods in randomized controlled trials. Sci Rep. 2017; 7:40837.

39. Streitberger K, Kleinhenz J. Introducing a placebo needle into acupuncture research. Lancet. 1998;352(9125):3645.

40. Deligdisch L. Hormonal pathology of the endometrium. Mod Pathol. 2000; 13(3):285-94.

41. Sponholtz TR, Palmer JR, Rosenberg LA, Hatch EE, Adams-Campbell LL, Wise LA. Exogenous hormone use and endometrial cancer in U.S. black women. Cancer Epidemiol Biomark Prev. 2018;27(5):558-65.

42. Demir B, Dilbaz S, Cinar O, Ozdegirmenci O, Dede S, Dundar B, et al. Estradiol supplementation in intracytoplasmic sperm injection cycles with thin endometrium. Gynecol Endocrinol. 2013;29:42-5.

43. Weckstein LN, Jacobson A, Galen D, Hampton K, Hammel J. Low-dose aspirin for oocyte donation recipients with a thin endometrium: prospective, randomized study. Fertil Steril. 1997;68:927-30.

44. Hsieh YY, Tsai HD, Chang CC, Lo HY, Chen CL. Low-dose aspirin for infertile women with thin endometrium receiving intrauterine insemination: a prospective, randomized study. J Assist Reprod Genet. 2000;17(3):174-7.

45. Gleicher N, Kim A, Michaeli T, Lee HJ, Shohat-Tal A, Lazzaroni E, et al. A pilot cohort study of granulocyte colony-stimulating factor in the treatment of unresponsive thin endometrium resistant to standard therapies. Hum Reprod. 2013;28:172-7

46. Kunicki M, Łukaszuk K, Liss J, Skowrońska P, Szczyptańska J. Granulocyte colony stimulating factor treatment of resistant thin endometrium in women with frozen-thawed blastocyst transfer. Syst Biol Reprod Med. 2017; 63:49-57.

47. Singh N, Mohanty S, Seth T, Shankar M, Bhaskaran S, Dharmendra S. Autologous stem cell transplantation in refractory Asherman's syndrome: a novel cell based therapy. J Hum Reprod Sci. 2014;7:93-8.

48. Kim H, Shin JE, Koo HS, Kwon H, Choi DH, Kim JH. Effect of autologous platelet-rich plasma treatment on refractory thin endometrium during the frozen embryo transfer cycle: a pilot study. Front Endocrinol. 2019;10:61.

49. Bos-Mikich A, de Oliveira R, Frantz N. Platelet-rich plasma therapy and reproductive medicine. J Assist Reprod Genet. 2018:35(5):753-6.

50. Ranisavljevic N, Raad J, Anahory T, Grynberg M, Sonigo C. Embryo transfer strategy and therapeutic options in infertile patients with thin endometrium: a systematic review. J Assist Reprod Genet. 2019;36:2217-31.

51. Takasaki A, Tamura H, Miwa I, Taketani T, Shimamura K, Sugino N. Endometrial growth and uterine blood flow: a pilot study for improving endometrial thickness in the patients with a thin endometrium. Fertil Steril. 2010;93(6):1851-8 
52. Lim HJ, Dey SK. HB-EGF: a unique mediator of embryo-uterine interactions during implantation. Exp Cell Res. 2009;315(4):619-26.

53. Cakmak H, Taylor HS. Implantation failure: molecular mechanisms and clinical treatment. Hum Reprod Update. 2011;17(2):242-53.

54. Craciunas L, Gallos I, Chu J, Bourne T, Quenby S, Brosens JJ, et al. Conventional and modern markers of endometrial receptivity: a systematic review and meta-analysis. Hum Reprod Update. 2019;25:202-23.

55. Remohí J, Ardiles G, García-Velasco JA, Gaitán P, Simón C, Pellicer A. Endometrial thickness and serum oestradiol concentrations as predictors of outcome in oocyte donation. Hum Reprod. 1997;12:2271-6.

56. Ng EH, Chan CC, Tang OS, Yeung WS, Ho PC. The role of endometrial blood flow measured by three-dimensional power Doppler ultrasound in the prediction of pregnancy during in vitro fertilization treatment. Eur J Obstet Gynecol Reprod Biol. 2007;135:8-16.

57. Silva MR, Helio OA, Vaz OD, Martinez de Oliveira J. Subendometrial resistence and pulsatility index assessment of endometrial receptivity in assisted reproductive technology cycles. Reprod Biol Endocrinol. 2019;17(1):62.

58. Serafini P, Batzofin J, Nelson J, Olive D. Sonographic uterine predictors of pregnancy in women undergoing ovulation induction for assisted reproductive treatments. Fertil Steril. 1994;62:815-22.

59. Riad ON, Hak AA. Assessment of endometrial receptivity using Doppler ultrasonography in infertile women undergoing intrauterine insemination. Gynecol Endocrinol. 2014;30(1):70-3.

60. Zhao J, Zhang Q, Wang Y, Li Y. Endometrial pattern, thickness and growth in predicting pregnancy outcome following 3319 IVF cycle. Reprod BioMed Online. 2014;29:291-8.

61. Shapiro H, Cowell C, Casper RF. The use of vaginal ultrasound for monitoring endometrial preparation in a donor oocyte program. Fertil Steril. 1993;59(5):1055-8

62. Achache H, Revel A. Endometrial receptivity markers, the journey to successful embryo implantation. Hum Reprod Update. 2006;12(6):731-46.

63. Paulson RJ. Hormonal induction of endometrial receptivity. Fertil Steril. 2011; 96(3):530-5.

64. Friedler S, Schenker JG, Herman A, et al. The role of ultrasonography in the evaluation of endometrial receptivity following assisted reproductive treatments: a critical review. Hum Reprod Update. 1996;2:323-35.

65. Teh WT, McBain J, Rogers P. What is the contribution of embryoendometrial asynchrony to implantation failure? J Assist Reprod Genet. 2016;33(11):1419-30

66. Cédrin-Durnerin I, Isnard T, Mahdjoub S, Sonigo C, Seroka A, Comtet M, et al. Serum progesterone concentration and live birth rate in frozenthawed embryo transfers with hormonally prepared endometrium. Reprod BioMed Online. 2019;38(3):472-80.

67. Shuai Z, Lian F, Li P, Yang W. Effect of transcutaneous electrical acupuncture point stimulation on endometrial receptivity in women undergoing frozenthawed embryo transfer: a single-blind prospective randomised controlled trial. Acupunct Med. 2015;33(1):9-15.

68. Ho M, Huang LC, Chang YY, Chen HY, Chang WC, Yang TC, et al. Electroacupuncture reduces uterine artery blood flow impedance in infertile women. Taiwan J Obstet Gynecol. 2009;48(2):148-51.

69. Qu F, Li R, Sun W, Lin G, Zhang R, Yang J, et al. Use of electroacupuncture and transcutaneous electrical acupoint stimulation in reproductive medicine: a group consensus. J Zhejiang Univ Sci B. 2017;18(3):186-93.

\section{Publisher's Note}

Springer Nature remains neutral with regard to jurisdictional claims in published maps and institutional affiliations.

Ready to submit your research? Choose BMC and benefit from:
- fast, convenient online submission
- thorough peer review by experienced researchers in your field
- rapid publication on acceptance
- support for research data, including large and complex data types
- gold Open Access which fosters wider collaboration and increased citations
- maximum visibility for your research: over 100M website views per year
At BMC, research is always in progress.
Learn more biomedcentral.com/submissions

